\title{
Peripheral Neurological Complications of Aortoiliac Vascular Disease
}

\author{
Monique L. D'Amour, Louise Hélène Lebrun, Antoine Rabbat, \\ Jules Trudel and Nicole Daneault
}

\begin{abstract}
Six patients with an aortoiliac vascular disease and a peripheral neurological deficit are presented. Clinical and electromyographic findings revealed lumbosacral plexus, sciatic and femoral nerve lesions. A correlation is made between the level of the vascular lesion (aortic, aortoiliac or distally) and the type of peripheral nerve deficit observed. In a patient complaining of pain, weakness, or numbness in a leg, the differential diagnosis should include aortoiliac vascular disease. The peripheral neurological symptoms may be the initial manifestation of the vascular disease or may appear in the early post-operative period.
\end{abstract}

\begin{abstract}
RÉSUMÉ: Complications neurologiques périphériques de la maladie vasculaire à localisation aorto-iliaque Cette étude décrit six malades qui ont présenté une maladie vasculaire aorto-iliaque et une atteinte neurologique périphérique des membres inférieurs. Leur déficit clinique et électromyographique a pu être localisé au niveau du plexus lombo-sacré, du nerf sciatique ou fémoral. Nous avons essayé d'établir une corrélation entre le déficit neurologique et le niveau d'atteinte vasculaire, soit aortique, aorto-iliaque ou distal. Les maladies vasculaires aorto-iliaques doivent être incluses dans le diagnostic étiologique d'une atteinte neurologique périphérique du membre inférieur. Cette atteinte neurologique périphérique peut être le mode de présentation de la maladie vasculaire ou se manifester dans les premiers jours après la chirurgie aorto-iliaque.
\end{abstract}

Can. J. Neurol. Sci. 1987; 14:127-130

Aortoiliac vascular disease is a common medical problem, but related neurological complications are not well described. A review of the literature reveals isolated case reports of spinal cord infarction, lumbar plexopathy or radiculopathy. In spinal cord infarction, the lesion is probably secondary to involvement of the major anterior radicular artery (artery of Adamkiewicz) ${ }^{\prime}$ originating from the thoracic or abdominal aorta. In cases of plexopathy or radiculopathy, the correlation between the vascular disease and the neurological complication is not so clearly documented.

We have had the opportunity of examining six patients suffering from aortoiliac vascular disease with peripheral lesions, either in the lumbosacral plexus or in the peripheral nerves. All patients were studied by means of an aortoiliac angiogram and underwent neurologic and electromyographic examinations. The radiological, clinical and neurophysiological findings were analyzed, and we attempted to establish a correlation between the vascular and neuroanatomical data to explain the neurological deficit observed.

\section{Clinical data}

The six patients were aged 45 to 67 and had no history of peripheral nerve disease. The first underwent surgery for an abdominal aortic aneurysm. The other five presented with complaints of claudication. Patients 3 and 6 also developed an acute pain in the right foot. Angiogram revealed atheromatous lesions at different levels of the aortoiliac axis. The type of surgery performed included aortofemoral bypass graft, femoropopliteal bypass, femoroiliac thrombectomy and endarterectomy.

A peripheral neurologic deficit was observed in two patients before surgery (cases 3 and 6) and in the remaining four patients in the days following surgery. Patient $I$ showed involvement of the right L2, L3, L4 roots or the upper part of the lumbar plexus. Patient 2 had more diffuse weakness suggesting a lesion involving roots $\mathrm{L} 2$ to $\mathrm{S} 1$ or the lumbosacral plexus. Patients 3,4 and 6 had more distal involvement from roots L4 to S2, or the distal distribution of the sciatic nerve, since the gluteal and quadriceps muscles were not involved. The hamstring muscles were unaffected in cases 3 and 6 and patient 5 had a lesion limited to the left femoral nerve distribution. Only one patient (case 4) had a bladder problem, consisting of urinary retention for two weeks not clearly related to the other symptoms.

An electromyogram (EMG) was performed on all patients one to six weeks after the onset of the neurological deficit. Signs of acute denervation with fibrillation and positive sharp waves were seen in five patients. In the other patient the recruitment pattern was reduced in muscles innervated by roots 
Table: Neurologic Findings, Antiographic Data and Surgical Procedures on Six Patients with Peripheral Neurological Complications of Aortoiliac Vascular Disease

\begin{tabular}{|c|c|c|c|}
\hline 4 & $\begin{array}{l}\text { R L4 to SI muscle weakness, sparing } \\
\text { gluteal and quadriceps muscles. } \\
\text { Hypoesthesia in L4, LS, SI distri- } \\
\text { bution. Decreased ankle jerk. }\end{array}$ & $\begin{array}{l}\mathrm{R} \text { common iliac stenosis } \\
\mathrm{R} \text { and } \mathrm{L} \text { internal iliac stenosis } \\
\mathrm{R} \text { superficial femoral occlusion } \\
\mathrm{L} \text { superficial femoral stenosis }\end{array}$ & $\begin{array}{l}\text { Aortofemoral bypass graft } \\
\mathrm{R} \text { femoral endarterectomy } \\
\mathrm{R} \text { femoropopliteal bypass }\end{array}$ \\
\hline
\end{tabular}

R: Right

L: Left

L2 to L4. The extent of the active denervation was more limited than the observed clinical weakness, probably because the EMG was performed early. Nerve conduction was either normal or showed abnormalities consistent with anoxal damage decreased amplitude of the evoked motor or sensory response and a slight slowing of nerve conduction velocity.

Patients 1, 2, 5 and 6 recovered over periods ranging from two weeks to a few months. Patients 3 and 4 still had residual motor deficits after one and three years respectively and patient 6 has residual pain in the leg. Each patient is described briefly in the following case reports and the data are summarized in the Table.

\section{Case Reports}

\section{Patient 1}

During a medical examination, an aneurysm of the abdominal aorta was discovered in this man who complained of vague abdominal discomfort. He had no neurological deficit and good pulses were felt in both feet. An angiogram showed marked dilatation of a $20 \mathrm{~cm}$ section of the abdominal aorta. The patient underwent surgery, which included clamping of the aorta, arteriotomy and evacuation of atheromatous material, ligation of the lumbar arteries and insertion of an aortobifemoral graft.

Proximal weakness in his right leg was noted the day following surgery. Good arterial pulses were found in both feet. Neurological examination on the fourth day revealed weakness of the right leg, which was moderate in the iliopsoas muscle and mild in the quadriceps and adductors. No bladder dysfunction was noted. The knee jerk was decreased. He had minimal hypoesthesia on the anterior aspect of the thigh. An EMG showed normal nerve conduction for peroneal, posterior tibial, femoral and sural nerves. Recruitment of motor units was reduced in the iliopsoas and quadriceps muscles.

\section{Patient 2}

This patient had had intermittent calf claudication for the past two years. On examination, femoral pulses were decreased and distal pulses were absent in both legs. There was no neurological deficit and no

bladder symptoms. An angiogram showed severe stenosis of the abdominal aorta. Stenosis was also present in the right common iliac together with occlusion of the right external iliac and left common iliac, and collateral flow distally from internal iliac arteries on both sides. An aortofemoral bypass graft was performed.

Two days after surgery, the patient noted weakness in his right leg. Neurological examination a week later revealed moderate weakness in the iliopsoas and adductor muscles, with mild weakness in the quadriceps, hamstring and anterior tibial muscles. The knee jerk was normal, the ankle jerk absent. He complained of pain over the anterior aspect of the thigh and diffuse numbness in the leg without obvious sensory deficit. Bladder function was normal. Arterial pulses were normal in both feet. An EMG performed two weeks later revealed normal peroneal, posterior tibial and sural nerve conduction. Fibrillations and positive sharp waves were seen in the anterior tibial and gastrocnemius muscles while the quadriceps, adductors, iliopsoas, gluteus medius and paraspinal L.3 to $\mathrm{S} 1$ muscles were spared.

\section{Patient 3}

This patient had undergone an aortobifemoral bypass three years previously for claudication. She came to the hospital after an acute pain in the right foot woke her up at night. On examination, the right leg was cold and pale below the knee. No pulses were detected in either leg. There was no dorsiflexion or plantar flexion of the foot and toes. Hypoesthesia to pain was noted below the knee. The angiogram showed occlusion of the right branch of the graft, $1 \mathrm{~cm}$ distal to the bifurcation. An iliofemoral thrombectomy was performed. Post-operatively, the foot was warm with no pulses. The patient made a gradual and partial recovery over the following months. Three weeks later an EMG revealed fibrillations and positive sharp waves in the tibialis anterior, gastrocnemius, tibialis posterior muscles but not, however, in the hamstring or gluteus medius. No responses could be elicited from stimulation of the peroneal, posterior tibial and sural nerves.

\section{Patient 4}

For several years this patient had suffered pain in both calves on walking. No pulses could be obtained distal to the femoral arteries. He had no neurological complaints. The angiogram showed moderate stenosis of the right common iliac and both internal iliac arteries, while the right femoral superficial artery was occluded and the left markedly stenotic. Distal vascularization came bilaterally from the deep femoral artery. An aortobifemoral bypass was done, in addition to right femoral endarterectomy and right femoropopliteal thrombectomy. 
Postoperatively, on the same day, he complained of pain in the right leg and could not move his foot, which was cold and pulseless. A femoropopliteal bypass was performed and full pulses were obtained. The following day, he started to complain of pain in the right buttock, the posterior aspect of the leg and the sole of the foot. The knee jerk was decreased, the ankle jerk absent. A few weeks later, weakness with fibrillation and positive sharp waves on EMG were noted in the extensor digitorum brevis, anterior tibial, gastrocnemius and abductor muscle of the great toe, while the quadriceps and gluteus medius were normal. Urinary retention lasted for two weeks. He subsequently made a slow recovery.

\section{Patient 5}

For the past year this man had complained of pain in both calves and thighs and the left buttock on walking. Pulses were absent at the femoral level and distally in both legs. The angiogram revealed moderate stenosis of both the common iliac and right external iliac arteries, with occlusion of left external iliac and both superficial femoral arteries. Collateral vascularization was seen distally in both legs. The patient underwent an abdominal aortic endarterectomy and aortobifemoral bypass graft. After surgery, the feet were warm but still pulseless. Two days later, he complained of pain over the anterior surface of the left thigh and the medial aspect of the left leg. Light touch and pinprick were diminished in that area. There was slight weakness of the quadriceps and iliopsoas muscles. The knee jerk was absent. An EMG carried out the following month revealed fibrillation and positive sharp waves in the quadriceps and iliopsoas muscles. Voluntary motor units could not be recruited in these muscles and no response could be evoked upon stimulation of the femoral nerve.

\section{Patient 6}

For the past six months, this patient had had intermittent claudication in both calves. During the past month, he had noted resting pain in the right foot. Suddenly, three weeks before admission, he noticed a sharp pain in the right calf and popliteal fossa and a burning sensation in the sole of the foot. Pulses were present at the femoral level but absent distally. There was an associated rubor in the right foot. No neurological deficit was observed. Angiography showed a right superficial femoral occlusion, moderate stenosis of the left superficial femoral and both common iliac arteries. A right femoropopliteal bypass with a saphenous vein and a superficial femoral endarterectomy were performed. In the days following the operation, pulses reappeared in the right foot. However, he complained of pain in the sole of the foot and the posterior aspect of the thigh. Hypoesthesia to pinprick was noted in the L5-S1 distribution and hyperesthesia over the sole. The ankle jerk was decreased. A few months later, an EMG revealed fibrillations and positive sharp waves in the gastrocnemius and extensor digitorum brevis. Polyphasic motor unit potentials were present in the anterior tibial muscle. The hamstrings, gluteus medius and gluteus maximus were normal. $\mathrm{He}$ made a partial recovery during the following year, but still complained of pain.

\section{Discussion}

The patients described in this study had a peripheral neurological deficit localized in the lumbosacral plexus, the sciatic or the femoral nerves. The common factor in all these patients was a vascular lesion of the aortoiliac segment, either proximal at the level of the aorta or more distal in the iliac arteries, or at both sites. Surgical procedures included resection of an abdominal aortic aneurysm, aortofemoral bypass graft, femoropopliteal bypass, thrombectomy or endarterectomy of the femoroiliac arteries.

Lumbar plexus lesions were seen in the first two patients. One had a resection of a large aneurysm of the abdominal aorta, ligation of lumbar arteries and an aortobifemoral graft. The second had severe stenosis of the abdominal aorta with external iliac occlusion, and underwent an aortofemoral bypass graft. Lumbosacral plexus lesions have been rarely reported as a neurological complication of aortoiliac vascular disease or surgery. Anatomically, the lumbosacral plexus runs deep and medial to the psoas muscle. Blood supply to this region includes, five lumbar arteries on each side of the abdominal aorta, the deep circumflex iliac artery, a branch of the external iliac, the iliolumbar and gluteal branches of the internal iliac artery. ${ }^{2.3}$ In cases of obstruction of the terminal aorta, the lumbar arteries may serve as the major collateral supply to the internal and external iliac arteries. Flow through the lumbar arteries may be impaired due to a vascular lesion of the aorta or they may be sacrificed during surgery involving clamping in a bypass procedure. These factors might explain the lumbar plexus lesions in patients 1 and 2 . One case of lumbosacral plexus and psoas muscle infarction was reported by Usubiaga et al ${ }^{4}$ after an aortobifemoral graft. Voulters and Bolton ${ }^{5}$ reported two cases of lumbosacral plexopathy, one following an aortofemoral bypass graft and the other after aortic manipulation by an intraortic balloon during surgery to replace a mitral valve. Ischemic monomelic neuropathy was reported by Wibourn et $\mathrm{al}^{6}$ in 14 patients; six were thromboembolic in origin and eight were due to various vascular surgical procedures. In one case denervation was found in the L4, L5, S1 distribution or the distal part of the sciatic nerve after a saddle embolus at the bifurcation of the aorta. In a chapter by Daube and Dyck, ${ }^{7}$ neuropathy secondary to occlusion of large arteries by embolism, thrombosis or arteriosclerotic occlusive disease has been reviewed. Lumbar radiculopathy was described as the initial clinical manifestation by Wilberger ${ }^{8}$ in three cases of abdominal aortic aneurysm. A case of lumbosacral plexus compression by a large aortoiliac aneurysm was reported by Giraldi. ${ }^{9}$

In three of our patients a lesion of the sciatic nerve seemed more likely than lumbosacral plexus involvement, since the gluteal and quadriceps muscles were not affected. This deficit has not been well documented in the literature. Gluteal artery aneurysms may compress the nerve. ${ }^{10}$ In these patients with probable sciatic nerve involvement, a vascular common denominator was a lesion of the common iliac artery. Impeded flow in the hypogastric artery (case 4) or in its origin from the common iliac artery (cases $3,4,6$ ), could explain eschemia of the sciatic nerve. Blood supply to this nerve is through the hypogastric or internal iliac artery. .,11.12 $^{2}$

Finally, in one patient (case 5), a lesion of the femoral nerve was found. Archie ${ }^{13}$ has described one case of femoral nerve involvement in a common iliac artery occlusion. This patient underwent an aortofemoral bypass. Nerve compression in the inguinal region could have been the mechanism involved, although ischemia due to clamping or embolization from the external iliac artery into the nerve might also have been contributing factors. The femoral nerve receives nutrient vessels from the iliolumbar, deep circumflex and lateral circumflex arteries, ${ }^{3}$ branches of internal, external and femoral arteries.

In two cases ( 3 and 6), neurological symptoms appeared during an acute or subacute vascular occlusion, just prior to vascular surgery. One patient woke up at night with an acute pain in the right leg. The angiogram on admission showed occlusion of the right branch of her previous aortofemoral graft, $1 \mathrm{~cm}$ distal to the bifurcation. Her leg showed signs of acute vascular insufficiency - it was cold, pale to the knee, and no pulses were present. The other patient had experienced progressive leg claudication over the previous six months, with resting pain in the right foot during the past month. He suddenly noted an acute pain in the right foot, calf and popliteal fossa. No 
pulses could be obtained in either leg. The angiogram showed occlusion of the right superficial femoral artery. Ischemia has to be considered as the pathophysiological factor not only in patients 3 and 6 , since symptoms appeared before surgery, but also for the other patients. Ischemia has been shown experimentally to produce Wallerian degeneration, demyelination ${ }^{14.15}$ or a physiological conduction block ${ }^{16}$ without morphological change, if it is of short duration. Ischemia without infarction could account for the rapid improvement in case 1 (two weeks). Alternatively, compression of the roots or nerves during surgery could be a possible cause of injury in this case. Nerve conduction block with relatively rapid recovery has been described in compression, with Wallerian degeneration being less important. ${ }^{17}$

We would like to emphasize the possibility of a peripheral neurological deficit in patients with aortoiliac vascular diseases. This should be considered when dealing with symptoms of leg pain and weakness at the time of a vascular occlusion or during the postoperative period. The vascular supply of the lower limb is through the aortoiliac tree, impairment of which might lead to ischemia of the various components of the limb including the nerves. Abdominal aortic lesions may result in lumbosacral plexus involvement, a common iliac artery lesion in sciatic nerve involvement, and lesion of the external iliac and superficial femoral arteries in femoral nerve involvement.

\section{REFERENCES}

I. Szilagyi DE, Hageman JH, Smith RF. Spinal cord damage in surgery of the abdominal aorta. Surgery $1978 ; 83: 38-56$.
2. Barker WF. Anatomy. In: Gaspar MR, Barker WF, eds. Peripheral arterial disease. WB Saunders Co., Philadelphia 1981; 1-37.

3. Sunderland $S$. Nerves and nerve injuries. Churchill Livingstone, Edinburgh 1978; 925, 961, 1000.

4. Usubiaga JE, Kolodny J, Usubiaga L. Neurological complications of prevertebral surgery under regional anesthesia. Surgery 1970 ; 68: 304-309.

5. Voulters L, Bolton C. Acute lumbosacral plexus neuropathy following vascular surgery. Can J Neurol Sci 1983; 10: 153.

6. Wilbourn A, Furlan AS, Hulley W, Ruschhaupt W. Ischemic monomelic neuropathy. Neurology 1983; 33: 447-451.

7. Daube JR, Dyck PJ. Neuropathy due to peripheral vascular disease. In: Dyck PJ, Thomas PK, Lambert EH, Bunge R. Peripheral neuropathy. WB Saunders Co., Philadelphia 1984; 1458-1478.

8. Wilberger JE. Lumbosacral radiculopathy secondary to abdominal aortic aneurysms. J Neurosurg 1983; 58: 965-967.

9. Giraldi C, Merlini V, Mosca F, Farrari M. Compressione del plesso lombo-sacrale in un caso di voluminoso aneurisma dell' arteria iliaca comune. Riv Neurol 1984; 54: 309-404.

10. Meek GN, Hill RL. Surgical treatment of gluteal artery aneurysms. Am J Surg 1968; 116: 731-734.

11. Anderson JE. Grant's Atlas of Anatomy. William \& Wilkins, Baltimore 1978; 7th Edition 4-12.

12. Sunderland S. Blood supply of the sciatic nerve and its popliteal divisions in man. Arch Neurol Psychiatry 1945; 283-289.

13. Archie JP. Femoral neuropathy due to common iliac artery occlusion. South Med J 1983; 76: 1073.

14. Eames RA, Lange LS. Clinical and pathological study of ischaemic neuropathy. J Neurol Neurosurg Psychiatry 1967; 30: 215-226.

15. Hess K, Eames RA, Darvenezia P, Gilliatt RW. Acute ischaemic neuropathy in the rabbit. J Neurol Sci 1979; 44: 19-43.

16. Parry GJ, Cornblath DR, Brown MJ. Transient conduction block following acute peripheral nerve ischemia. Muscle and Nerve 1985; 8: 409-412.

17. Rudge P, Ochoa J, Gilliat RW. Acute peripheral nerve compression in the baboon. J Neurol Sci 1974; $23: 403-420$. 